\title{
A Rare and Fatal Co-Occurrence of Evans Syndrome and Autoimmune Hepatitis/Primary Biliary Cirrhosis Overlap Syndrome
}

\section{Kolipakkam B* and Vachhani H}

Department of Internal Medicine, Virginia Commonwealth University, USA

*Corresponding author: Bharadhwaj Kolipakkam, Department of Internal Medicine, Virginia Commonwealth University, Richmond, USA, Tel: 8572108990; Email: bharadhwajk@gmail.com

\section{Case Report}

Volume 4 Issue 1

Received Date: April 06, 2020

Published Date: April 24, 2020

DOI: $10.23880 /$ hij-16000157

\section{Abstract}

Evans syndrome (ES) is the concurrent occurrence of two or more lines of immune-mediated cytopenias commonly involving autoimmune hemolytic anemia (AIHA) and immune thrombocytopenia (ITP). It can be an idiopathic condition or may be associated with lymphoproliferative and autoimmune disorders. Here we report a case of a person with a history of Autoimmune Hepatitis (AIH) and Primary Biliary Cirrhosis (PBC) Overlap Syndrome, on chronic steroids, who presented with thrombocytopenia and autoimmune hemolytic anemia and diagnosed with Evans syndrome. This case brings forth the difficulty in diagnosing ES, especially in patients with liver disease who can have overlapping laboratory abnormalities and the poor prognosis associated with the condition, despite treatment with IVIg and immunomodulatory agents, like rituximab.

Keywords: Evans Syndrome; Immunomodulatory agents; Autoimmune hepatitis; Ulcerative colitis

Abbreviations: ES: Evans Syndrome; AIHA: Autoimmune Hemolytic Anemia; ITP: Immune Thrombocytopenia; AIH: Autoimmune Hepatitis; PBC: Primary Biliary Cirrhosis; SLE: Systemic Lupus Erythematosus.

\section{Introduction}

Initially described in 1951, Evans syndrome (ES) is the concurrent occurrence of two or more lines of immunemediated cytopenias commonly involving autoimmune hemolytic anemia (AIHA) and immune thrombocytopenia (ITP); Immune neutropenia can rarely occur. ES is a rare condition because it is diagnosed in only $0.8 \%$ to $3.7 \%$ of all patients with either ITP or AIHA. Here we report a case of ES in Autoimmune Hepatitis (AIH) and Primary Biliary Cirrhosis (PBC) Overlap Syndrome.

\section{Case Presentation}

A 46-year-old man with a past medical history of AIH/ PBC overlap syndrome (on chronic steroids) and ulcerative colitis was admitted with thrombocytopenia $(9,000$ cells/ $\mathrm{mm}^{3}$ ), lower than his baseline of 30,000 . Given that there was no response to platelet transfusion, this raised the suspicion for Immune Thrombocytopenia. He was started on IV Immunoglobulin (IVIg) with improvement in platelet count to baseline and was discharged on an increased dose of prednisone. However, he presented a week later with fatigue, nausea, and vomiting. A review of systems was notable for dark-colored urine and negative for fever, chills, and abdominal pain. The examination was significant for severe jaundice and pallor; no hepatosplenomegaly or ascites was noted. Laboratory investigation was significant for macrocytic anemia (hematocrit 15\%), reticulocyte index $2.7 \%$, LDH $1200 \mathrm{U}$ and peripheral smear showed large platelets with no schistocytes. Direct Coombs was positive and haptoglobin was undetectable. Total bilirubin was increased to $50 \mathrm{mg} / \mathrm{dl}$ with indirect bilirubin of $20 \mathrm{mg} / \mathrm{dl}$. Given these findings, a diagnosis of Evans syndrome was made, and he was restarted on IVIg along with high dose steroids. In the absence of adequate response, Rituximab was started for refractory disease. Unfortunately, his clinical condition deteriorated with acute liver failure and multi- 
organ dysfunction and the patient expired the following day [1-3].

\section{Discussion}

Although ES was initially considered to be an idiopathic condition, it may be associated with systemic lupus erythematosus (SLE), lymphoproliferative and other immune disorders.- Our patient had an autoimmune milieu which likely played a role. This case brings forth the difficulty in diagnosing ES, especially in patients with liver disease who can have overlapping laboratory abnormalities. The inappropriate response to transfusion and response to IVIg and steroids favors immune-mediated destruction of platelets and RBCs. Corticosteroid and/or IVIG therapy seem to be effective as first-line treatment in patients with ES. Second-line therapy is usually immunosuppressive drugs or Rituximab. Prognosis tends to be worse in patients with ES than with AIHA or ITP alone [4,5].

\section{Conclusion}

In our extensive literature search, only one case of ES in AIH/PBC overlap syndrome has been reported thus far, making this case extremely rare. A high degree of suspicion is required to diagnose this condition as treatment modalities differ compared to other causes.

\section{References}

1. Jaime-Pérez JC, Aguilar-Calderón PE, Salazar-Cavazos L, Gómez-Almaguer D (2018) Evans syndrome: clinical perspectives, biological insights and treatment modalities. J Blood Med 9: 171-184.

2. Korkmaz H, Bugdaci MS, Temel T, Dagli M, Karabagli $\mathrm{P}$ (2013) Autoimmune hepatitis-primary biliary cirrhosis overlap syndrome concomitant with immune hemolytic anemia and immune thrombocytopenic purpura (Evans syndrome). Clin Res Hepatol Gastroenterol 37(2): 45-50.

3. Azad A, Berera V, Jayarajan J, Lim K (2007) Evans syndrome and primary biliary cirrhosis. Int J Lab Hem 29(2): 145-148.

4. Wang W, Herrod H, Pui CH, Presbury G, Willimas J (1983) Immunregulatory abnormalities in Evans syndrome. Am J Hematol 15(4): 381-390.

5. Michel M, Chanet V, Dechartres A, Morin AS, Piette JC, et al. (2009) The spectrum of Evans syndrome in adults: new insight into the disease based on the analysis of 68 cases. Blood 114(15): 3167-3172. 